\title{
Characterization of 3-Dimensional PET Systems for Accurate Quantification of Myocardial Blood Flow
}

\author{
Jennifer M. Renaud ${ }^{1}$, Kathy Yip ${ }^{2}$, Jean Guimond ${ }^{3}$, Mikaël Trottier ${ }^{3}$, Philippe Pibarot ${ }^{3}$, Eric Turcotte , Conor Maguire $^{5}$, \\ Lucille Lalonde $^{5}$, Karen Gulenchyn ${ }^{6}$, Troy Farncombe ${ }^{6}$, Gerald Wisenberg ${ }^{7}$, Jonathan Moody ${ }^{8}$, Benjamin Lee ${ }^{8}$, \\ Steven C. Port ${ }^{9}$, Timothy G. Turkington ${ }^{10}$, Rob S. Beanlands ${ }^{1}$, and Robert A. deKemp ${ }^{1}$ \\ ${ }^{I}$ National Cardiac PET Centre, University of Ottawa Heart Institute, Ottawa Ontario, Canada; ${ }^{2}$ KMH Cardiology \& Diagnostic \\ Centre, Mississauga Ontario, Canada; ${ }^{3}$ Institut Universitaire de Cardiologie et de Pneumologie de Québec, Québec, Canada; ${ }^{4}$ Centre \\ Hospitalier Universitaire de Sherbrooke, Québec, Canada; ${ }^{5}$ University of Alberta Hospital, Edmonton Alberta, Canada; ${ }^{6}$ St. Joseph's \\ Healthcare, Hamilton Ontario, Canada; ${ }^{7}$ Lawson Health Research Institute, London Ontario, Canada; ${ }^{8}$ INVIA Medical Imaging \\ Solutions, Ann Arbor, Michigan; ${ }^{9}$ Aurora Cardiovascular Services, Milwaukee, Wisconsin; and ${ }^{10}$ Duke University Medical Center, \\ Durham, North Carolina
}

Three-dimensional (3D) mode imaging is the current standard for $\mathrm{PET} / \mathrm{CT}$ systems. Dynamic imaging for quantification of myocardial blood flow with short-lived tracers, such as ${ }^{82} \mathrm{Rb}$-chloride, requires accuracy to be maintained over a wide range of isotope activities and scanner counting rates. We proposed new performance standard measurements to characterize the dynamic range of PET systems for accurate quantitative imaging. Methods: ${ }^{82} \mathrm{Rb}$ or ${ }^{13} \mathrm{~N}-$ ammonia (1,100-3,000 MBq) was injected into the heart wall insert of an anthropomorphic torso phantom. A decaying isotope scan was obtained over 5 half-lives on 9 different 3D PET/CT systems and $13 \mathrm{D} / 2$-dimensional PET-only system. Dynamic images $(28 \times 15 \mathrm{~s})$ were reconstructed using iterative algorithms with all corrections enabled. Dynamic range was defined as the maximum activity in the myocardial wall with less than $10 \%$ bias, from which corresponding dead-time, counting rates, and/or injected activity limits were established for each scanner. Scatter correction residual bias was estimated as the maximum cavity blood-to-myocardium activity ratio. Image quality was assessed via the coefficient of variation measuring nonuniformity of the left ventricular myocardium activity distribution. Results: Maximum recommended injected activity/body weight, peak dead-time correction factor, counting rates, and residual scatter bias for accurate cardiac myocardial blood flow imaging were 3-14 MBq/kg, 1.5-4.0, 22-64 Mcps singles and 4-14 Mcps prompt coincidence counting rates, and $2 \%-10 \%$ on the investigated scanners. Nonuniformity of the myocardial activity distribution varied from $3 \%$ to $16 \%$. Conclusion: Accurate dynamic imaging is possible on the 10 3D PET systems if the maximum injected $\mathrm{MBq} / \mathrm{kg}$ values are respected to limit peak dead-time losses during the bolus first-pass transit.

Key Words: dynamic range; cardiac positron emission tomography; ${ }^{82} \mathrm{Rb}$

J Nucl Med 2017; 58:103-109

DOI: 10.2967/jnumed.116.174565

\footnotetext{
Received Mar. 18, 2016; revision accepted Jul. 17, 2016.

For correspondence or reprints contact: Robert deKemp, Room $\mathrm{H}-1215$, National Cardiac PET Centre, University of Ottawa Heart Institute, Ottawa Ontario, Canada.

E-mail: radekemp@ottawaheart.ca

Published online Aug. 18, 2016.

COPYRIGHT (C 2017 by the Society of Nuclear Medicine and Molecular Imaging.
}

$\mathbf{P}$ ET imaging in 3-dimensional (3D) mode has become the standard for new whole-body scanners. The increased sensitivity allows for reduction of injected activity to the patient while maintaining excellent image quality; however, random and scattered photon counts are increased, requiring systems with high counting rate capability and accurate corrections for these physical effects. Current PET instrumentation and National Electrical Manufacturers Association (NEMA) performance evaluation methods (1) have been developed primarily to optimize whole-body oncology imaging with ${ }^{18}$ F-FDG. However, dynamic PET imaging for myocardial blood flow (MBF) quantification with short-lived tracers, such as ${ }^{82} \mathrm{Rb},{ }^{15} \mathrm{O}$-water, or ${ }^{13} \mathrm{~N}$-ammonia, requires high counting rates and correction accuracy to be maintained over a wide range of measured activities (2). An ideal PET system should allow for conventional relative myocardial perfusion imaging (MPI) of tracer retention without compromising accuracy of first-pass dynamic data (3). Routine MBF imaging is clinically feasible with the 76-s half-life generator-produced tracer ${ }^{82} \mathrm{Rb}$, resulting in accurate $(4,5)$ and reproducible measurements $(3,6-8)$, as validated against ${ }^{13} \mathrm{~N}$-ammonia and ${ }^{15} \mathrm{O}$-water standards $(9-12)$.

We proposed methods to evaluate the dynamic operating range of 3D PET systems for quantitative imaging of MBF. Patient imaging protocols were implemented and used to confirm the predicted operating range.

\section{MATERIALS AND METHODS}

\section{Phantom Scans}

Image Acquisition. ${ }^{82} \mathrm{Rb}$ decaying isotope scans were obtained over 5 half-lives using an anthropomorphic torso phantom (model ECT/ TOR/P; Data Spectrum Corp.), approximating a small male upper torso $(38 \times 26 \mathrm{~cm})(13)$ on 8 different 3D PET/CT systems and $13 \mathrm{D} / 2$ dimensional (2D) PET-only system. An ${ }^{13} \mathrm{~N}$-ammonia scan was obtained on 1 other 3D PET/CT camera (Supplemental Table 1; supplemental materials are available at http://jnm.snmjournals.org). The phantom contained a myocardial heart cavity and wall insert (model ECT/CAR/I; Data Spectrum Corp.), lungs, spine, and liver chamber (Figs. 1A and 1B). Liver and body cavities were filled with water to mimic soft-tissue attenuation. The phantom was placed in the prone position in the PET field of view to facilitate infusion directly into the myocardial wall, and $1,500-3,000 \mathrm{MBq}(40-80 \mathrm{mCi})$ of ${ }^{82} \mathrm{Rb}$ or $1,100 \mathrm{MBq}$ of ${ }^{13} \mathrm{~N}$-ammonia were infused. ${ }^{82} \mathrm{Rb}$ was infused either as a 30 -s square-wave with saline 


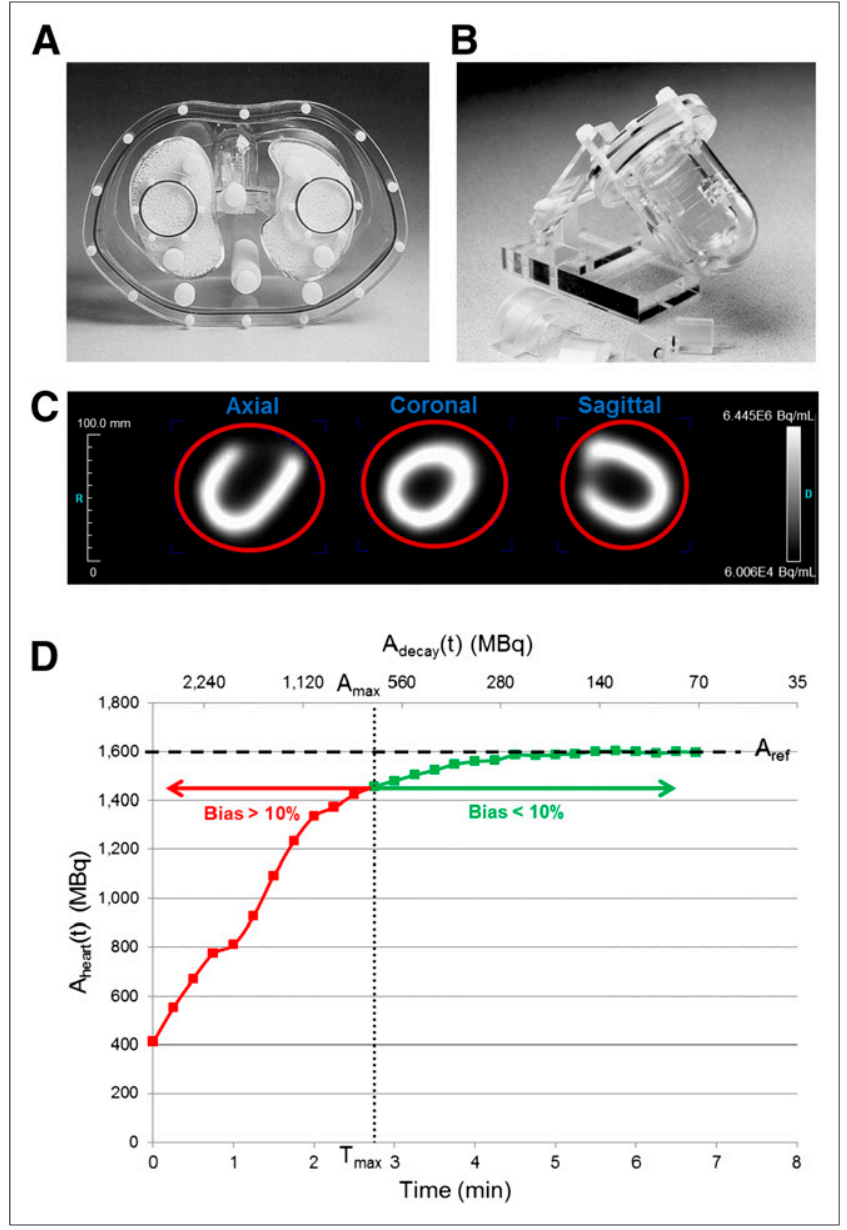

FIGURE 1. Anthropomorphic torso phantom (A), including cardiac insert (B), simulating a small male patient. Volume of interest (red) (C) drawn over entire cardiac insert and resultant time-activity curve (D). Dashed (horizontal) line indicates reference activity value $\left(A_{\text {reff }}\right)$. Dotted (vertical) line denotes threshold $\left(A_{\max }, T_{\max }\right)$ between accurate and inaccurate quantitative values.

push or as a $50 \mathrm{~mL} / \mathrm{min}$ bolus (Supplemental Table 1). A list-mode PET acquisition was started immediately after completion of tracer infusion, simulating the localized activity and high counting rate observed during tracer first-pass transit through the heart. For attenuation correction, the PET scan was followed by a low-dose CT scan on the PET/CT systems or a 4-min transmission scan on the PET-only camera.

Image Reconstruction. Dynamic images (28 frames $\times 15 \mathrm{~s}$ ) were reconstructed using vendor-supplied Fourier rebinning-filtered backprojection or iterative expectation-maximization algorithms (14), with an 8or 12-mm Hann or Gaussian postprocessing filter and all corrections enabled for isotope decay, attenuation, scatter, randoms, prompt-gammas, detector efficiency, and dead-time, according to routine clinical practice at each institution. Most systems had explicit prompt $\gamma$-correction enabled during reconstruction (Supplemental Table 1); others used a 50-cm CT attenuation correction field of view to minimize the contribution of prompt $\gamma$-photons to the 3D coincidence background (15).

Quantitative Analysis. Reconstructed image time-activity curves were analyzed to determine the dynamic operating range for which quantitative accuracy was maintained. Total injected activity time-activity curves were measured using Inveon Research Workplace software (Siemens) (Figs. 1C and 1D). A spheric volume of interest (10-cm diameter) encapsulating the activity in the heart insert was drawn (Fig. 1C) from which total decay-corrected activity, $\mathrm{A}_{\text {heart }}(\mathrm{t})(\mathrm{MBq})$, was measured for all mid-frame scan times, $\mathrm{t}$ (min). From the time-activity curve, the average decay-corrected activity in the late time frames, where tracer uptake had reached a stable maximum, was determined as the true reference value, $A_{\text {ref }}(\mathrm{MBq})$. Activity bias in each time frame was then calculated as:

$$
\operatorname{Activity} \operatorname{bias}(\mathrm{t})=\left(\mathrm{A}_{\text {heart }}(\mathrm{t}) / \mathrm{A}_{\text {ref }}-1\right) \times 100(\%) .
$$

To compare dynamic range among scanners, Activity bias (t) was plotted as a function of total activity in the heart volume of interest, $\mathrm{A}_{\text {decay }}(\mathrm{t})$, where:

$$
\mathrm{A}_{\text {decay }}(\mathrm{t})=\mathrm{A}_{\text {ref }} \times \mathrm{e}^{-\lambda \mathrm{t}}(\mathrm{MBq})
$$

For ${ }^{82} \mathrm{Rb}$ and ${ }^{13} \mathrm{~N}$-ammonia, the isotope decay constants are $\lambda=\ln (2) /$ 1.27 and $\ln (2) / 10$ (min), respectively. The time, $T_{\max }$, of the earliest frame with $10 \%$ or less activity bias was identified (Fig. 1D), and total heart activity at $\mathrm{t}=\mathrm{T}_{\max }$ was calculated as:

$$
A_{\max }=A_{\text {heart }}\left(T_{\max }\right) \times \mathrm{e}^{-\lambda T \max }(\mathrm{MBq}) .
$$

Dead-time correction factors (DTFs) and prompt coincidences and/or singles counting rates associated with the highest activity, $A_{\max }$, were tabulated as available in the image headers. The maximum weight-based activity recommended for patient studies was estimated as $\mathrm{A}_{\max } \mathrm{di}$ vided by $50 \mathrm{~kg}$, the representative body weight of the torso phantom, determined according to the attenuating cross-sectional area, which is approximately 2.3 times larger than the NEMA scatter phantom previously shown to represent a $21.5-\mathrm{kg}$ patient (16). A repeated scan was obtained on the Discovery 690, 600, and the Biograph PET/CT-16 systems to assess reproducibility of injected activity/body weight values.

Scatter correction residual bias was estimated as the left ventricular (LV) cavity blood-to-myocardium ratio by plotting scatter bias(t) as a function of $\mathrm{A}_{\text {decay }}(\mathrm{t})$, where:

$$
\text { Scatter bias }(\mathrm{t})=\left(\mathrm{C}_{\text {cavity }}(\mathrm{t}) / \mathrm{C}_{\text {myo }}(\mathrm{t})\right) \times 100(\%) \text {. }
$$

$\mathrm{C}_{\text {cavity }}(\mathrm{t})$ represents average activity concentration in the heart cavity $\left(\mathrm{Bq} / \mathrm{cm}^{3}\right)$, and $\mathrm{C}_{\mathrm{myo}}(\mathrm{t})$ is the average concentration in the myocardial wall $\left(\mathrm{Bq} / \mathrm{cm}^{3}\right)$. Residual bias is an indicator of uncorrected scatter in the LV cavity and is important to measure because accurate scatter correction is required for quantitative MBF measurements using an imagederived input function. To extract myocardium and LV cavity blood time-activity curves, our in-house FlowQuant software was used (3). The blood-to-myocardium ratio was determined by taking the median of the cavity, base, and atrium time-activity curves and then dividing by the myocardium average time-activity curve.

Image quality was assessed as nonuniformity of the myocardium activity distribution, using the coefficient of variation (COV) of the LV polar map:

$$
\mathrm{C}_{\mathrm{myo}}(\mathrm{t})_{\mathrm{COV}}=\mathrm{SD}_{\mathrm{myo}}(\mathrm{t}) / \mathrm{C}_{\mathrm{myo}}(\mathrm{t}) \times 100(\%),
$$

where $\mathrm{SD}_{\text {myo }}(\mathrm{t})$ is the $\mathrm{SD}$ of the activity concentration in the myocardial wall polar map. Images were also inspected visually for count ratedependent pile-up artifacts.

\section{Patient Scans}

Patient Population. Recommended weight-based activity and DTF limits defined by the phantom scans were validated using ${ }^{82} \mathrm{Rb}$ PET images from 20 patients acquired on the Discovery 690 and 600 and the Scintron 3D PET cameras (Supplemental Table 2). All patients were referred for a clinically indicated myocardial perfusion scan 
for coronary artery disease diagnosis or risk stratification. The institutional review board (or equivalent) at each of the participating centers approved this study, and all subjects signed a written informed consent form.

Image Acquisition. On the Discovery cameras, the ${ }^{82} \mathrm{Rb}$ rest scan was followed by a dipyridamole stress scan, whereas regadenoson stress was used on the Scintron. Injected activity of $10 \mathrm{MBq} / \mathrm{kg}$ of body weight was prescribed for patients scanned on the Discovery systems, and $8 \mathrm{MBq} / \mathrm{kg}$ on the Scintron, according to local clinical practice for MPI. At rest and stress, 6-min list-mode acquisitions were started at the time of injection to capture the first-pass transit of the tracer as required for MBF quantification (Supplemental Fig. 1).

Quantitative Analysis. DTFs were tabulated for each time frame to identify the peak counting rates and dead-time losses. Global LV MBF values were computed automatically using FlowQuant, as described for phantom scans. Blood and LV myocardium time-activity curves were used as input to a 1-tissue-compartment model with a constant distribution volume to estimate $\operatorname{MBF}(4,6)$.

\section{Statistical Analysis}

Values are presented as mean $\pm \mathrm{SD}$. Where applicable, means were compared via the Student $t$ test or 1-way ANOVA using SPSS Statistics 23 (IBM). A $P$ value of less than 0.05 was considered statistically significant.

\section{RESULTS}

\section{Phantom Scans}

Figure 1D shows the bias in measured activity as a function of time $(t)$ and total activity in the heart phantom insert, $A_{\text {decay }}(t)$, for a single scanner. The $\mathrm{A}_{\max }$ with less than $10 \%$ bias was $325 \mathrm{MBq}$. Assuming a representative phantom mass of $50 \mathrm{~kg}$, the highest recommended patient-equivalent injected activity/body weight was estimated as $6.5 \mathrm{MBq} / \mathrm{kg}$. At this activity (frame 12 at $2.75 \mathrm{~min}=$ $\mathrm{T}_{\text {max }}$ ), the peak prompt and singles counting rates and DTF were 4.1 and 29 Mcps and 2.0, respectively (Table 1).

Across all investigated scanners, the maximum recommended injected activity/body weight, peak DTF, and counting rate for accurate dynamic, quantitative cardiac MBF imaging varied between
3 and 14.4 MBq/kg, 1.5-4.0 DTF, and 22-64 Mcps singles and 4-14 Mcps prompt counting rate, respectively (Table 1). As expected, scanners using optimized detector crystals (higher atomic number, shorter decay time, higher light output (17)) and/or improved processing electronics were found to accommodate higher injected activity/body weight while remaining quantitatively accurate (Fig. 2). Peak DTF values within the accurate range corresponded typically with peak coincidence dead-times of $50 \%$ or less. Peak counting rates varied considerably between scanners, and intercomparison was not possible in all cases, depending on the camera-specific information available. Repeated scans were within $4 \% \pm 9 \%$ of the originally tabulated injected activity/body weight values (Discovery 690, 13.1 MBq/kg [+6.8\%]; Discovery 600, 5.7 MBq/kg [-6.4\%]; and Biograph PET/CT-16, 6.9 MBq/kg [+11.0\%]), demonstrating good reproducibility of the proposed methodology.

Residual scatter bias varied from $2 \%$ to $10 \%$ within the accurate operating range (Table 1). Highly variable uncorrected scatter was observed for all scanners in early time frames when counts tend to pile up toward the center of the detector blocks (Fig. 3A). Within the accurate operating range only, scatter bias stabilized at a relatively constant level (Fig. 3B). This bias was found to be slightly higher on the lutetium oxyorthosilicate detector-based systems $(7.8 \pm 2.0)$ versus the other scanners $(2.9 \pm 1.1 ; P<0.05)$, suggesting that the scatter-correction methods implemented on these 4 lutetium oxyorthosilicate-based scanners may benefit from further optimization to improve accuracy.

All phantom images showed high contrast and low noise over the entire range of activity. Assessment of the LV myocardium polar map nonuniformity demonstrated that COV was highest (lowest image quality) in early frames $\left(\mathrm{t}=0-\mathrm{T}_{\max }, \mathrm{C}_{\mathrm{myo}}(\mathrm{t})_{\mathrm{COV}}=10.2 \% \pm 4.9 \%\right)$ (Fig. 4A). COV values stabilized within the recommended operating range $\left(\mathrm{t}=\mathrm{T}_{\max }-7 \mathrm{~min}, \mathrm{C}_{\mathrm{myo}}(\mathrm{t})_{\mathrm{COV}}=8.9 \% \pm 3.4 \% ; P=\right.$ not significant vs. early frames) (Fig. 4B), corresponding with the trend observed for residual scatter bias. These results suggest that despite high dead-time losses in the early time frames, image quality is not compromised and is not a limiting factor for

TABLE 1

Recommended Maximum Injected Activity and Performance Metrics

\begin{tabular}{|c|c|c|c|c|c|c|}
\hline PET system & $\begin{array}{c}\text { Patient } A_{\max } / \text { weight } \\
(\mathrm{MBq} / \mathrm{kg})\end{array}$ & $\begin{array}{l}\text { Peak prompts } \\
\text { (Mcps) }\end{array}$ & $\begin{array}{l}\text { Peak singles } \\
\text { (Mcps) }\end{array}$ & $\begin{array}{l}\text { Peak } \\
\text { DTF }\end{array}$ & $\begin{array}{l}\text { Scatter bias }(\mathrm{t}) \\
(\%)\end{array}$ & $\begin{array}{c}\mathrm{C}_{\mathrm{myo}}(\mathrm{t})_{\mathrm{cov}} \\
(\%)\end{array}$ \\
\hline Biograph mCT PET/CT-40 & 14.4 & 6.3 & 64 & - & $5.2 \pm 0.2$ & $12.4 \pm 2.1$ \\
\hline ECAT Accel Scintron PET 2D & 11.4 & 1.6 & 26 & 1.7 & $8.3 \pm 0.6$ & $6.5 \pm 0.2$ \\
\hline Discovery 690 PET/VCT-64 & 11.4 & 5.9 & 45 & 1.5 & $2.4 \pm 0.3$ & $11.0 \pm 4.8$ \\
\hline Discovery IQ (5 ring) PET/CT-16 & 11.3 & 14.1 & 84 & 3.9 & $2.7 \pm 1.1$ & $2.9 \pm 0.2$ \\
\hline Biograph TruePoint PET/CT-16 & 8.0 & - & - & - & $9.9 \pm 0.4$ & $8.0 \pm 0.3$ \\
\hline Discovery 600 PET/CT-16 & 6.5 & 4.1 & 29 & 2.0 & $2.1 \pm 0.3$ & $12.1 \pm 1.1$ \\
\hline Biograph PET/CT-16 & 5.5 & - & 22 & - & $8.6 \pm 0.2$ & $16.4 \pm 1.3$ \\
\hline Discovery RX PET/CT-16 & 5.1 & 4.5 & - & 1.7 & $3.1 \pm 0.3$ & $10.6 \pm 0.8$ \\
\hline Gemini TF PET/CT-16 & 4.6 & - & - & - & $2.5 \pm 0.5$ & $7.8 \pm 0.9$ \\
\hline Discovery STE/VCT-16 & 3.9 & 3.5 & - & 2.1 & $2.5 \pm 0.4$ & $4.3 \pm 3.3$ \\
\hline ECAT Accel Scintron PET 3D & 2.7 & 1.6 & 22 & 1.7 & $7.4 \pm 0.2$ & $6.5 \pm 0.3$ \\
\hline
\end{tabular}

$-=$ Not available in image header files. $\mathrm{t}=\mathrm{T}_{\max }$ to $7 \mathrm{~min}$. 


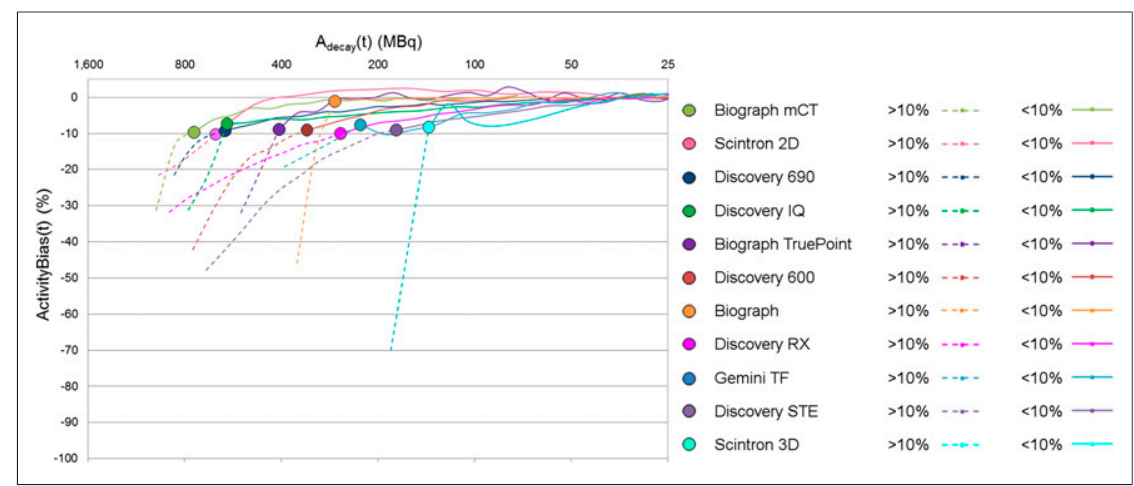

FIGURE 2. Total heart activity, $A_{\text {decay }}(t)(M B q)$, versus activity bias(t) (\%). Dotted lines denote activity greater than $10 \%$ bias, whereas solid lines represent activity less than $10 \%$ bias. The highest activity with $10 \%$ bias or less, $A_{\max }$ (circles), indicates maximum amount of activity that can be injected while maintaining quantitatively accurate values.

quantitative accuracy. Visual image inspection confirmed the absence of any obvious counting rate-dependent pile-up artifacts for all PET systems.

\section{Patient Scans}

Delivered activity was $10.3 \pm 0.3$ and $9.9 \pm 2.0 \mathrm{MBq} / \mathrm{kg}$ for patients imaged on the Discovery 690 and 600 systems. Peak DTF values were $1.5 \pm 0.1$ and $2.1 \pm 0.2$ (corresponding to $33 \%$ and $50 \%$ coincidence dead-time, respectively), similar to the recommended maxima suggested by the phantom scans (Table 1). For patients scanned on the Scintron with $8 \mathrm{MBq} / \mathrm{kg}$, peak DTF was $1.6 \pm 0.2$ (38\% coincidence dead-time), slightly lower than the phantom maximum value recommended to remain within the accurate dynamic operating range.

\section{DISCUSSION}

This study established methods to evaluate the accurate dynamic operating range of 3D PET systems for quantitative cardiac imaging with ${ }^{82} \mathrm{Rb}$. Decaying isotope phantom scans were obtained over 5 half-lives to determine the optimal operating range, defined by the maximum injected activity/body weight, and corresponding maximum singles, prompt coincidence counting rates, or peak dead-time factors. Patient scans were obtained near the suggested limits on 3 representative scanners and confirmed validity of the phantom scan recommendations. Evaluation of the scatter correction bias

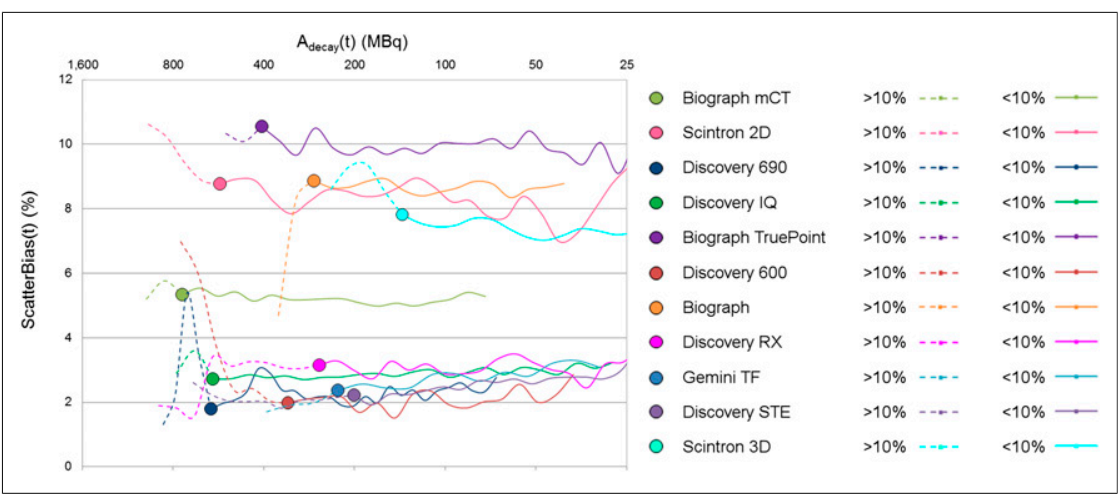

FIGURE 3. LV cavity blood-to-myocardium ratio plots of residual scatter. In early frames where activity is high enough to saturate detectors (activity bias $>10 \%$ ) (dotted lines), there is highly variable residual scatter, which stabilizes in the accurate range of operation ( $<10 \%$ bias) (solid lines). confirmed the effectiveness of manufacturerimplemented scatter corrections in 3D mode. Finally, LV polar map nonuniformity, and the absence of counting rate-dependent pile-up artifacts, was found to be adequate for diagnostic evaluation.

The results suggest that the evaluated PET scanners should be able to perform accurate quantitative imaging despite differences in manufacturing technology, including scintillation detectors, detector block size, coincidence processing hardware, and prompt- $\gamma$-correction availability. The most important factor to consider for quantitative imaging in patients is that peak dead-time, singles, and/or prompt coincidence counting rates remain below the threshold values determined from the phantom scans to obtain accurate images and prevent a biased MBF estimation. This technique allows for prospective determination of image accuracy, as opposed to retrospective evaluation of detector block saturation or other performance metrics after acquisition is completed (18). It can also be performed retrospectively as long as counting rate and/or dead-time parameters are contained in the image header files generated by the scanners. As opposed to the NEMA counting rate performance standard that is designed for whole-body oncology imaging, the proposed method measures myocardial activity using a more realistic cardiac imaging geometry, and the residual scatter fraction and myocardial image uniformity are measured at the highest counting rates typically encountered during the bolus first pass.

Scan header information obtained from most of the scanners did not include complete counting rate and dead-time information, which would make it impossible to retrospectively verify that patient scans were obtained within the accurate operating range, and therefore that quantitative MBF values were absolutely reliable. It would be beneficial if all manufacturers recorded this information in the scan headers to allow consistent evaluation of quantitative imaging performance.

\section{Clinical Implications}

An optimal PET imaging system should allow for conventional MPI and absolute MBF imaging with a single injection of tracer. For PET cameras with adequate dynamic range and for which the injected activity/ body weight limit is high enough to obtain diagnostic-quality perfusion images, both static MPI and dynamic MBF images can be obtained with a single injection protocol. In our previous multicenter study, $10 \mathrm{MBq} / \mathrm{kg}$ was the benchmark established for diagnostic-quality MPI using 3D PET systems (15). If this threshold was applied to the cameras in the current study, a single injection protocol could be recommended on the first 4 systems listed in Table 1; the others would require a dual-injection protocol to first obtain diagnostic-quality perfusion images using a high-dose injection and then accurate images for MBF assessment with a lower-dose scan. However, all 


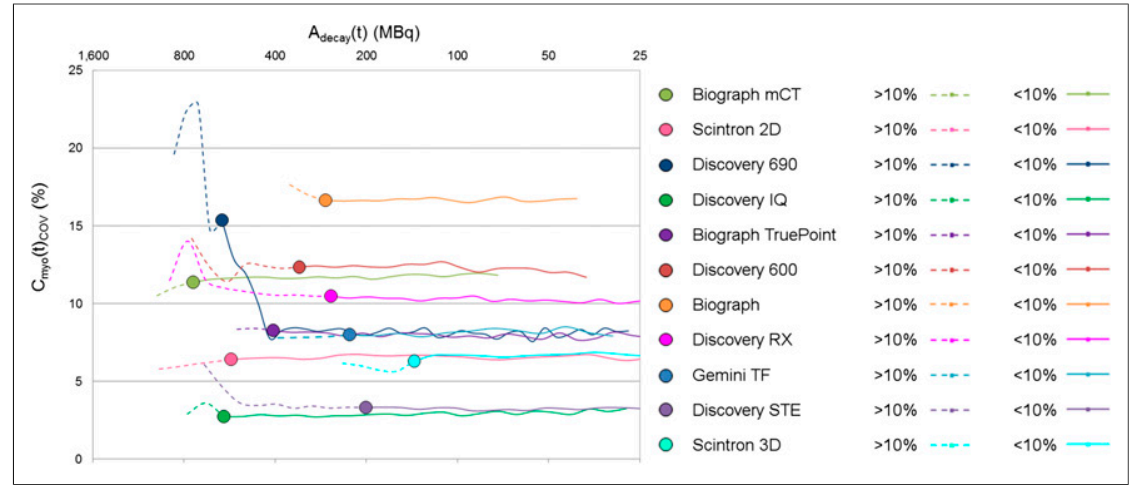

FIGURE 4. LV myocardium polar map nonuniformity (COV). Outside the accurate operating range (bias $>10 \%$ in early frames), COV is highly variable (dotted lines), whereas it reaches a relatively constant level within the accurate operating range (solid lines).

systems showed maintained image quality in the early frames outside of the accurate range, as measured by COV, suggesting that the dynamic range may be extended with vendor improvements in dead-time correction accuracy, potentially allowing for a single-injection protocol on additional systems.

If a single-injection protocol is used on a system without sufficient dynamic range, MBF values would be inaccurate because peak blood and myocardium activities would be underestimated due to high dead-time losses at activity values exceeding the dynamic range of the camera. A patient example demonstrating this effect is shown in Figure 5. A 170-cm, 100-kg female patient was scanned at rest on the Discovery 600 PET/CT system with an injected dose of $10 \mathrm{MBq} / \mathrm{kg}$ of ${ }^{82} \mathrm{Rb}$, above the maximum limit for accurate quantification determined by the phantom scan, and a 5-fold-lower dose of $2 \mathrm{MBq} / \mathrm{kg}$, for comparison. At $10 \mathrm{MBq} / \mathrm{kg}$, the peak DTF and prompt counting rates were 2.5 and $6.8 \mathrm{Mcps}$, exceeding the recommended limits of 2.0 and $4.1 \mathrm{Mcps}$, suggesting that the camera was not operating in the accurate dynamic range for that portion of the scan. As a result, the early peak values of the blood and LV myocardium time-activity curves are underestimated compared with the low-dose time-activity curves (Fig. 5A). Underestimation of the area under the blood curve causes overestimation of

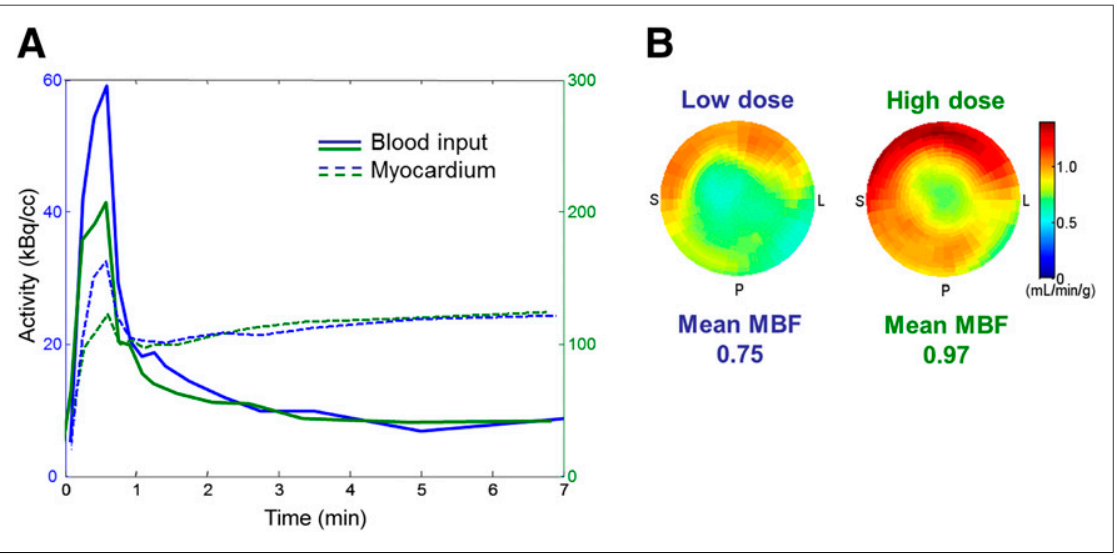

FIGURE 5. MBF results for patient scanned on Discovery 600 PET/CT system. (A) Blood and myocardium time-activity curves for injected activity/weight of $2 \mathrm{MBq} / \mathrm{kg}(227 \mathrm{MBq} / 101 \mathrm{~kg})$ (blue) and $10 \mathrm{MBq} / \mathrm{kg}$ (1022 MBq/101 kg) (green). At $10 \mathrm{MBq} / \mathrm{kg}$, peak blood and myocardium activities are underestimated, (B) resulting in overestimation of LV myocardium MBF values. $(S, P$, and $L$ denote septal, posterior and lateral LV walls.) the MBF values, as shown in the LV myocardium polar maps (Fig. 5B).

\section{Patient Scan Variability}

To estimate the maximum weight-based activity to use for patient studies, $A_{\max }$ was divided by the representative weight of the phantom, estimated as $50 \mathrm{~kg}$. A scannerdependent estimate based on DTF values obtained using a particular activity/body weight protocol may be more appropriate. The patient data required to perform this estimate were available for the Discovery 690, 600, and Scintron 3D. The peak DTF values from the patient scans vary according to body weight (Fig. 6A), therefore for each scanner the phantom DTF curve was plotted as a function of the weight-based dose $(\mathrm{MBq} / \mathrm{kg})$, using representative phantom weights corresponding with (passing through) the mean and maximum patient DTF values (Fig. 6B). Adjustment of the representative phantom weight improves prediction of the recommended injected dose for a range of patient sizes and also accounts for differences in absolute calibration between the PET scanner and rubidium elution system (used to measure the phantom and patient injected activities, respectively). The difference in calibration was relatively small $(-6 \%$ to $+20 \%)$ between the RUBY-FILL (Jubilant DraxImage Inc.) elution system and Discovery scanners, whereas the difference was much larger $(-68 \%)$ between the CardioGen82 (Bracco Diagnostics Inc.) infuser and Scintron scanner. On the basis of the combined results, slightly lower injected activities of 9, 7, and $6 \mathrm{MBq} / \mathrm{kg}$ for the Discovery 690, 600, and Scintron would be required to keep all patient DTF values within the accurate operating range. For other scanners, the recommended injected activity/body weight values should be confirmed or adjusted using similar methods.

\section{Comparison with Previous Studies}

In previous work by Tout et al. (18), the dynamic range of the Biograph mCT for simultaneous ${ }^{82} \mathrm{Rb}$ MPI and MBF assessment was investigated in patients. They determined that a dose of $1,110 \mathrm{MBq}(30 \mathrm{mCi})$ resulted in lower detector block saturation (1\%) versus the manufacturer-recommended dose of $1,480 \mathrm{MBq}(40 \mathrm{mCi})(15 \%$ saturation). All patients were injected with the same activity rather than the weight-based dosing proposed here. When the weightbased value of $14.4 \mathrm{MBq} / \mathrm{kg}$ determined in the present study for this scanner model and the mean weight of $87 \mathrm{~kg}$ from the population studied in Tout et al. were used, an average injected activity of $1,250 \mathrm{MBq}$ would be recommended, similar to their suggested value of 1,110 MBq. As we have previously presented (19), administered activity can be adjusted for patient weight to compensate for the tracer distribution volume in the body and increased attenuation. Diagnostic image quality would likely be improved with higher doses in larger 


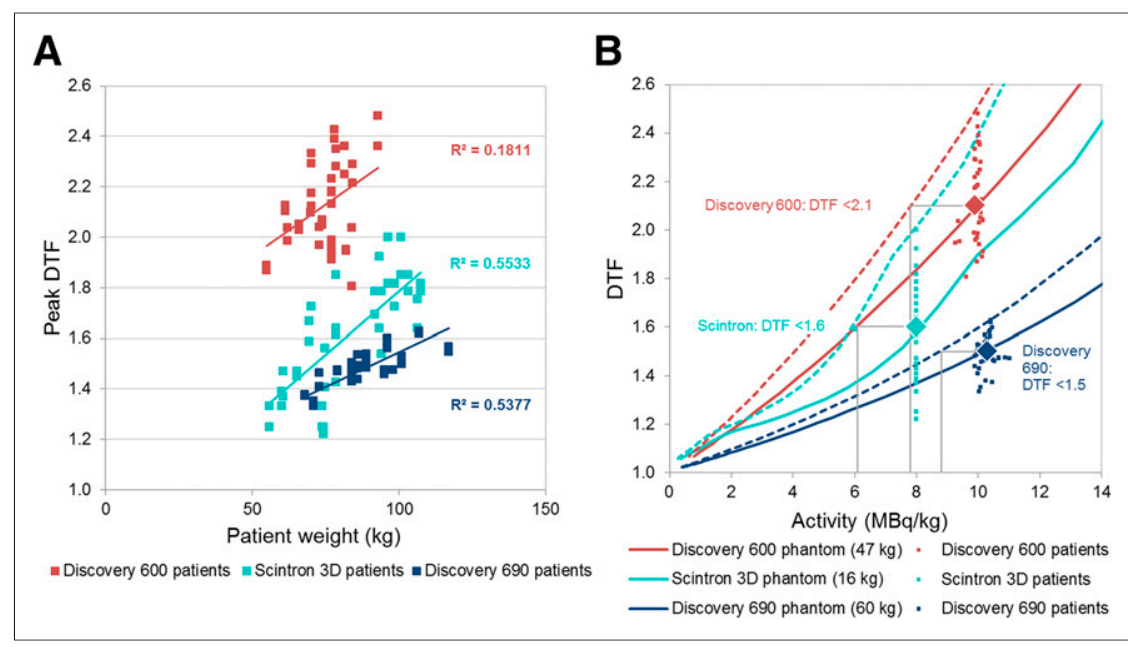

FIGURE 6. (A) Patient peak DTF varies with body weight. (B) Similarly, peak (first-pass) DTFs increase as a function of injected activity/weight for phantom scans (solid lines). Using clinical MPI doses of $10 \mathrm{MBq} / \mathrm{kg}$ for patients on Discovery 690 and 600 systems and $8 \mathrm{MBq} / \mathrm{kg}$ on Scintron, mean DTF values ( $1.5 \pm 0.1,2.1 \pm 0.2,1.6 \pm 0.2$; large diamonds) agreed with recommended phantom-determined limits. Adjusted phantom curves (dashed lines) show that to ensure all patient scans remain below maximum recommended peak DTF values, lower injected activities would be required (gray lines). Wider DTF distribution in patients on the Discovery 600 and Scintron may reflect higher randoms rates measured with bismuth germanium oxide and early generation lutetium oxyorthosilicate detectors, compared with lutetium-based scintillator detectors on the 690 . As activity increases, bismuth germanium oxide systems produce much higher, more variable, random coincidences because of the wider coincidence time window.

patients, whereas smaller patients would benefit from lower radiation dose with maintained image quality. Most importantly in smaller patients, a standard dose may exceed the scanner dynamic range during the bolus first pass and prevent accurate MBF quantification. The method of Tout et al. relies on verifying detector block saturation post hoc, which is not possible in real-time during patient scanning and is a more complex procedure than simple observation of the peak DTF or system counting rates during the patient scan.

Recently, Kolthammer et al. (20) investigated the dynamic range of the Ingenuity $\mathrm{TF}$, the successor to the Gemini TF evaluated in the present study. A cylinder phantom was infused with 4 separate doses of ${ }^{82} \mathrm{Rb}$, ranging from 370 to $1,480 \mathrm{MBq}$, with a 10-min PET acquisition starting simultaneously with the infusion. Dynamic images were reconstructed into 15-s time frames. From this experiment, they determined that ${ }^{82} \mathrm{Rb}$ imaging was accurate up to a peak singles counting rate of $65 \mathrm{Mcps}$ at an injected activity of $925 \mathrm{MBq}$. For an average-sized patient of $70 \mathrm{~kg}$, $925 \mathrm{MBq}$ corresponds to an injected activity/body weight of approximately $13 \mathrm{MBq} / \mathrm{kg}$. For the Gemini TF investigated in the present study, we obtained a maximum recommended activity of approximately $5 \mathrm{MBq} / \mathrm{kg}$, suggesting that the Ingenuity TF may accommodate higher injected activities due to improved detector crystals (lutetium-yttrium oxyorthosilicate) and electronics. Because the singles counting rates were not stored in the Gemini TF header files, these values could not be compared with the Ingenuity TF scanner.

In another recent study, O'Doherty et al. (21) investigated the effect of scanner dead-time on MBF values obtained from kinetic modeling of ${ }^{13} \mathrm{~N}$-ammonia dynamic images acquired on the Discovery 710 PET/CT scanner. They showed that global LV MBF values in 4 patients were $8.9 \% \pm 0.6 \%$ higher when the
LV blood-pool input function was corrected for high dead-time losses in the early frames using the percentage difference between measured versus true activity obtained from phantom studies. These preliminary results again demonstrate the importance of using appropriate cameraspecific maximum injected activity levels to obtain accurate MBF assessments.

\section{CONCLUSION}

Dynamic imaging to obtain accurate quantitative MBF measurements with ${ }^{82} \mathrm{Rb}$ appears feasible on the 10 3D PET systems evaluated when the recommended peak dead-time, maximum counting rates, and injected activity limits are respected. $\mathrm{Pa}$ tient scans confirmed the validity of the injected activity/body weight recommendations to achieve accurate and reliable quantitative images.

\section{DISCLOSURE}

This study was funded by Canadian Institute of Health Research grant MIS100935 (Rb-ARMI). Jennifer M. Renaud and Robert A. deKemp receive royalties from FlowQuant ${ }^{\circledR}$ sales. Robert A. deKemp receives royalties from rubidium $\mathrm{PET}$ technology licenses. Rob S. Beanlands and Robert A. deKemp received grant funding from a government/industry research program (Ontario Research Fund/ Industry Partners: GE Healthcare, Nordion, Lantheus Medical Imaging, and Jubilant DraxImage Inc.). Jennifer M. Renaud, Rob S. Beanlands, and Robert A. deKemp are consultants for Jubilant DraxImage Inc. Rob S. Beanlands is a consultant for Lantheus Medical Imaging and a career scientist, supported by the Heart and Stroke Foundation of Ontario, Vered Chair of Cardiology and University of Ottawa Tier 1 Chair in Cardiovascular Imaging Research. Timothy G. Turkington is a consultant for Data Spectrum Corporation and has received grant funding from GE Healthcare. No other potential conflict of interest relevant to this article was reported.

\section{ACKNOWLEDGMENTS}

We thank the technologists and research staff at the participating sites for their invaluable efforts in acquiring the data discussed herein. We acknowledge Jubilant DraxImage Inc. for provision of the ${ }^{82} \mathrm{Rb}$ generators, elution system, training, and support.

\section{REFERENCES}

1. National Electrical Manufacturers Association. Performance Measurements of Positron Emission Tomographs. NEMA standards publication NU2-2012. Rosslyn, VA: NEMA; 2013.

2. deKemp RA, Yoshinaga K, Beanlands RSB. Will 3-dimensional PET-CT enable the routine quantification of myocardial blood flow? J Nucl Cardiol. 2007;14: 380-397.

3. Klein R, Renaud JM, Ziadi MC, et al. Intra- and inter-operator repeatability of myocardial blood flow and myocardial flow reserve measurements using 
rubidium-82 pet and a highly automated analysis program. J Nucl Cardiol. 2010;17:600-616.

4. Lortie M, Beanlands RS, Yoshinaga K, et al. Quantification of myocardial blood flow with ${ }^{82} \mathrm{Rb}$ dynamic PET imaging. Eur J Nucl Med Mol Imaging. 2007;34:1765-1774.

5. Prior JO, Allenbach G, Valenta I, et al. Quantification of myocardial blood flow with ${ }^{82} \mathrm{Rb}$ positron emission tomography: clinical validation with ${ }^{15} \mathrm{O}$-water. Eur J Nucl Med Mol Imaging. 2012;39:1037-1047.

6. Efseaff M, Klein R, Ziadi MC, Beanlands RS, deKemp RA. Short-term repeatability of resting myocardial blood flow measurements using Rb-82 PET imaging. J Nucl Cardiol. 2012;19:997-1006.

7. DeKemp RA, Declerck J, Klein R, et al. Multisoftware reproducibility study of stress and rest myocardial blood flow assessed with 3D dynamic PET/CT and a 1-tissue-compartment model of ${ }^{82} \mathrm{Rb}$ kinetics. J Nucl Med. 2013;54: 571-577.

8. Nesterov SV, Deshayes E, Sciagra R, et al. Quantification of myocardial blood flow in absolute terms using ${ }^{82} \mathrm{Rb}$ PET imaging: the RUBY-10 Study. JACC Cardiovasc Imaging. 2014;7:1119-1127.

9. Katoh C, Yoshinaga K, Klein R, et al. Quantification of regional myocardial blood flow estimation with three-dimensional dynamic rubidium-82 PET and modified spillover correction model. J Nucl Cardiol. 2012;19:763-774.

10. Yoshinaga K, Manabe O, Katoh C, et al. Quantitative analysis of coronary endothelial function with generator-produced ${ }^{82} \mathrm{Rb}$ PET: comparison with ${ }^{15}$ O-labelled water PET. Eur J Nucl Med Mol Imaging. 2010;37:2233-2241.

11. Renaud JM, DaSilva JN, Beanlands RS, deKemp RA. Characterizing the normal range of myocardial blood flow with ${ }^{82}$ rubidium and ${ }^{13} \mathrm{~N}$-ammonia PET imaging. J Nucl Cardiol. 2013;20:578-591.
12. El Fakhri G, Kardan A, Sitek A, et al. Reproducibility and accuracy of quantitative myocardial blood flow assessment with ${ }^{82} \mathrm{Rb}$ PET: comparison with ${ }^{13} \mathrm{~N}$-ammonia PET. J Nucl Med. 2009;50:1062-1071.

13. Anthropomorphic Torso Phantom ${ }^{\mathrm{TM}}$ specification sheet. Data Spectrum Corporation website. http://www.spect.com/pub/Anthropomorphic_Torso_Phantom.pdf. Updated June 29, 2008. Accessed October 21, 2016.

14. Hudson HM, Larkin RS. Accelerated image reconstruction using ordered subsets of projection data. IEEE Trans Med Imaging. 1994;13:601-609.

15. Renaud JM, Mylonas I, McArdle B, et al. Clinical interpretation standards and quality assurance for the multicenter PET/CT trial rubidium-ARMI. J Nucl Med. 2014;55:58-64.

16. Watson CC, Casey ME, Bendriem B, et al. Optimizing injected dose in clinical PET by accurately modeling the counting-rate response functions specific to individual patient scans. J Nucl Med. 2005;46:1825-1834.

17. Melcher CL. Scintillation crystals for PET. J Nucl Med. 2000;41:1051-1055.

18. Tout D, Tonge CM, Muthu S, Arumugam P. Assessment of a protocol for routine simultaneous myocardial blood flow measurement and standard myocardial perfusion imaging with rubidium-82 on a high count rate positron emission tomography system. Nucl Med Commun. 2012;33:1202-1211.

19. Klein R, Beanlands RSB, deKemp RA. Quantification of myocardial blood flow and flow reserve: technical aspects. J Nucl Cardiol. 2010;17:555-570.

20. Kolthammer JA, Su KH, Grover A, Narayanan M, Jordan DW, Muzic RF. Performance evaluation of the Ingenuity TF PET/CT scanner with a focus on high count-rate conditions. Phys Med Biol. 2014;59:3843-3859.

21. O' Doherty J, Schleyer P, Pike L, Marsden P. Effect of scanner dead time on kinetic parameters determined from image derived input functions in ${ }^{13} \mathrm{~N}$ cardiac PET. J Nucl Med. 2014;55(suppl 1):605. 